\title{
Automatic motor cortex activation for natural as compared to awkward grips of a manipulable object
}

Received: 26 December 2004 / Accepted: 20 May 2005 / Published online: 22 July 2005

(C) Springer-Verlag 2005

\begin{abstract}
It has been suggested that, relative to natural objects, man-made object representations in the brain are more specifically defined by functional properties that reflect how an object is used and/or what it is used for (Warrington and Shallice 1984). We recorded 123channel event-related potentials (ERP) in healthy participants during a mental rotation task involving a manipulable (hammer) and a non-manipulable (church) object. Both stimuli had standard and mirror-image versions rotated in four different orientations, resulting for the manipulable object in some natural and some awkward grips. Using spatial cluster analysis, time periods were determined during which the ERP maps differed between stimulus conditions. Specific maps appeared for natural versus awkward grips with the manipulable object at a very early stage $(60-116 \mathrm{~ms})$ as well as during a later stage $(180-280 \mathrm{~ms})$. Source estimations derived from the topographic data indicated that during the second time window the left motor cortex was significantly activated in the case of natural grips. We argue that the motor programs that are
\end{abstract}

L. S. Petit $(\square) \cdot$ I. M. Harris

Macquarie Centre for Cognitive Science, Macquarie University, Sydney, NSW 2109, Australia

E-mail: leila.petit@epfl.ch

Tel.: + 41-21-693-16-59

Fax: +41-21-693-96-25

C. M. Michel · L. S. Petit

Functional Brain Mapping Laboratory, Neurology Clinic, University Hospital and Department of Basic Neuroscience, University Medical School,

1211 Geneva, Switzerland

A. J. Pegna

Centre for Cognitive Neuroscience, School of Psychology, University of Wales, Bangor, UK

L. S. Petit

Laboratory of Cognitive Neuroscience

and Laboratory of Psychophysics,

Brain-Mind Institute,

Ecole Polytechnique Fēdérale de Lausanne,

Swiss Federal Institute of Technology,

1015 Lausanne, Switzerland semantically associated with the object are automatically activated when it is presented in graspable orientations.

Keywords Mental rotation - Manipulable object . ERP mapping $\cdot$ Electric source imaging

\section{Introduction}

Humans are particularly efficient at representing the world and themselves internally. It is not necessary to execute an action in order to know its consequences because anticipation is possible by mental representation. There is evidence that imagined spatial transformations of body parts are strongly influenced by their normal range of motion (Parsons 1987; Petit et al. 2003; Petit and Harris in press). In this study, we were interested in mental imagery of tools since they are manmade objects created to imitate or extend the function of a body segment, but without its biomechanical constraints. However, tools possess specific functional properties and the question arises whether these are taken into account during mental representations and transformations.

It is argued that visual object characteristics may help to elicit an action associated with an object and Gibson (1979) referred to the concept of affordance to describe the fact that we perceive objects in terms of the ways in which they can be used. There is evidence provided by behavioural studies suggesting an automatic activation of action representations when objects are perceived. Indeed, several authors have investigated the existence of an automatic link between the visual perception of an object and a specific predetermined action. For instance, Craighero et al. (1996) showed that a prime, visually congruent with an object to be grasped, significantly reduces grasping reaction time (RT). Tucker and Ellis (1998) studied the relation between an object's orientation and the best-suited hand to reach and grasp it. They observed faster right-hand responses when the object 
orientation was compatible with a right-hand grasp, and similarly for the left-hand responses. Moreover, De'Sperati and Stucchi (1997) presented subjects with a rotating screwdriver and asked them whether it was screwing or unscrewing. They showed that, in righthanders, stimulus orientations that were particularly awkward for a right-hand grip resulted in higher response times compared with visually equivalent, but more comfortable orientations. Thus, there seems to be an automatic use of motor imagery to mentally simulate a movement of the preferred hand, consequently combining visual cues with procedural motor knowledge. Grèzes et al. (2003) investigated the influence of intrinsic properties of an object on motor responses that were either compatible, or incompatible with the action afforded by the object. Their results showed faster motor responses to objects that afforded a congruent grip. In a previous study on the mental rotation of a hammer (Petit et al. 2003), we found a significant difference in RTs between the natural and awkward grips even though the tool had the same angular departure from the upright. We expected to find similar results for the same angles departing from the upright clockwise (natural grip) or counter clockwise (awkward grip), but found longer RTs for the counter clockwise angles. The distinction between natural and awkward postures for the hammer turned out to be relevant for such an object and was interpreted as the result of an internal process that links the object with the functional properties of the actions that are carried out with it. Finally, Tucker and Ellis (2004) showed that the on-line presence of a visual object is not necessary to prime a particular type of grasp. Even with exposure times well below $100 \mathrm{~ms}(30$ and $50 \mathrm{~ms}$ ) objects induced affordance compatibility effects, despite not being visible at the time the response was selected, prepared and executed.

These results support the hypothesis that visually presented tools automatically activate components of the actions they afford, even in the absence of explicit intentions to act. Motor theories of perception (Viviani and Stucchi 1992) claim that certain classes of perceptual facts are based on an internal model of motor acts. Motor imagery is a mental activity in itself, but it also underlies some non-motor functions: perceptual processes tap motor information that remains available independently from any intention by the perceiver to act.

Evidence supporting this view comes from studies in monkeys. Rizzolatti et al. (1988) discovered that within the ventral premotor area F5 "canonical neurons" not only become active when the monkey grasps an object but also when it looks at the object in the absence of any subsequent motor activity. Taira et al. (1990) showed that neurons of the anterior intraparietal (AIP) sulcus share similar functional properties with those of F5. Thus, some neurons in the AIP sulcus (Taira et al. 1990) and a region in ventral premotor cortex area F5 (Rizzolatti et al. 1988) discharge when the monkey looks at graspable objects (Sakata et al. 1995). Hence, Jeannerod et al. (1995) suggested that the AIP-F5 network is responsible for transforming the intrinsic properties of objects into the appropriate hand movements and that actions associated with graspable objects are automatically evoked whenever the monkey sees these objects. Since tools are usually associated with specific hand movements, it seems that perceiving manipulable objects is closely related to information about the actions that are carried out with them and that this perception activates a neuronal network which includes motor knowledge. Following the same line of thinking, Murata et al. (1997) suggested that the description of the object's properties is first carried out in the parietal area AIP and then transferred to the premotor area F5 to select a potential action.

Many neuroimaging studies investigated the neural basis of those canonical neurons in humans and hence investigated the nature and characteristics of action representations while perceiving an object. For instance, Chao and Martin (2000) compared various categories of objects in an fMRI study that revealed a selectivity of the left ventral premotor cortex for pictures of tools. In a previous study, the same authors (Chao et al. 1999) showed that pictures of tools elicited bilateral activity in the medial aspect of the fusiform gyrus and the middle temporal gyrus. They concluded that all these regions form a network that links information about the visual characteristics and properties of tools with the appropriate distal movements necessary for using them and which is active whenever we recognize and identify manipulable man-made objects. These findings suggest that functional properties are important for identifying tools that are strongly associated with specific hand movements. In a PET study, Grafton et al. (1997) presented subjects with real tools of common use that they had to observe. This task activated the left dorsal premotor cortex in an area where arm/hand movements are represented as well as a region between area 45 and area 46. Finally, in an fMRI study, Grèzes et al. (2003) found activation of parietal and premotor areas when subjects observed manipulable objects. Thus, common activations have been found in premotor and posterior parietal cortex for monkeys and humans.

Together, these results are consistent with the hypothesis that imagery of tools is influenced by their functional characteristics, one of them being that there is motor involvement in visual representation of objects. Thus, there is a large body of evidence that the observation of an object merely to identify it is sufficient to activate motor representations. What remains undetermined at present are the temporal dynamics of brain activation during the representation of objects such as tools. Is the motor cortex activated simultaneously with perceptive areas or subsequently? If it is a serial process, how long after initial perception do the motor mechanisms become involved? If motor processes occur at a very early stage (i.e., in parallel with perceptive processes), this would imply that they are actually an essential component of forming representations of objects. However, if these motor processes appear at a later 
stage (i.e., subsequently to perception), when subjects are already aware of the object, they could simply be a by-product of object representation. In the present study, we sought to advance our understanding of the neural processes and temporal dynamics of brain activation associated with intrinsic manipulability, by investigating the mental rotation of a manipulable object, which is a paradigm that does not imply an intention to act. Subjects had to perform a classical mental rotation task, i.e., to determine whether two pictures of the same object were identical or mirror images. A hammer represented the manipulable object and within its various orientations, some of them were presented in a "ready-for-use" orientation for right-handers and were thus considered to be "more manipulable" (or natural) than others (more awkward orientations). As far as behavioural results were concerned, we expected to find longer RTs for the less manipulable orientations of the hammer.

Since we were interested in the sequence of brain processes involved during the task, we used event-related potential (ERP) recordings, a technique that has a millisecond time resolution. We applied spatio-temporal analysis techniques to the data to search for different processing patterns underlying the recognition processes of different orientations, such as those depicting natural versus awkward orientations of the manipulable object, and those requiring mental rotation.

\section{Methods}

\section{Participants}

Thirteen healthy university-level subjects (eight males, five females) took part in this study. All were between 24 and 32 years of age (mean 28 years). Handedness was evaluated using the Oldfield-Edinburgh questionnaire (Oldfield 1971). All participants were considered righthanded (laterality index range: +80 to +100 ) and all had normal or corrected-to-normal vision. Participants gave their written informed consent after having received instructions concerning the experimental procedure.

\section{Stimuli}

The test stimulus consisted of a picture of a manipulable object (hammer) taken from the Premium Image Collection of Hemera Technologies Inc. (1997-2000) and transformed with a picture-editing software (Corel Photo-Paint).

A standard and a mirror-image version of the picture were created (see Fig. 1a), and these were presented in four orientations: $0^{\circ}, 90^{\circ}, 180^{\circ}$ and $270^{\circ}$.

The control stimulus was a non-manipulable object (a church), using exactly the same experimental paradigm as for the hammer. Here again, a standard and a
$\mathrm{R}$

T)

b

$\mathrm{R}$

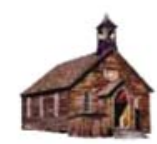

$\mathrm{L}$

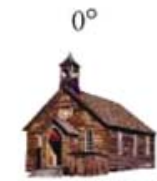

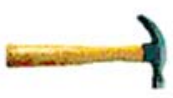

$90^{\circ}$
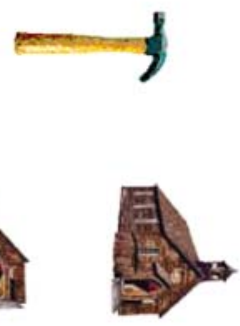

$90^{\circ}$

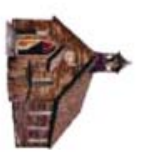

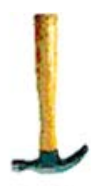

$180^{\circ}$
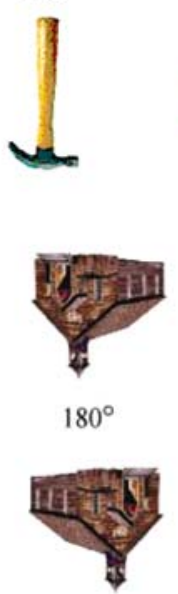
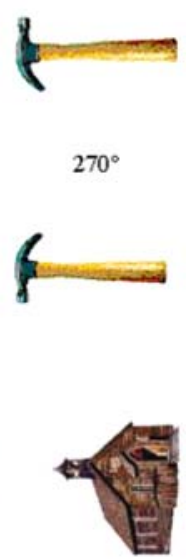

$270^{\circ}$

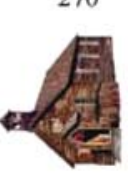

Fig. 1 Illustration of the stimuli used. For the test stimulus (a), the hammer, $0^{\circ}$ and $270^{\circ}$ depict natural grip orientations and $90^{\circ}$ and $180^{\circ}$ depict awkward grip orientations. For both the test (a) and the control (b) stimuli, the top line represents the object in the posture facing right $(R)$ and the bottom line represents the posture facing left $(L)$

mirror-image version of the picture were created (see Fig. 1b), and these were presented in the same four orientations as the hammer: $0^{\circ}, 90^{\circ}, 180^{\circ}$ and $270^{\circ}$.

\section{Procedure}

For both the test and the control experiment, each trial consisted of a picture presented for $250 \mathrm{~ms}$ at the centre of the screen, always in the upright (i.e., $0^{\circ}$ ) orientation, followed by a $700 \mathrm{~ms}$ blank screen, followed by a second picture, which was presented for $250 \mathrm{~ms}$ at the same location as the first picture, but in various orientations. A fixation cross then appeared lasting 2,450 ms during which the subject gave his/her response. The subjects' task was to determine whether the two stimuli were identical or mirror images. RTs were recorded from the second picture onset time to the key-press. If no response was given in $3 \mathrm{~s}$, the following trial was administered.

The pictures of both experiments were presented in two different blocks. However, the pictures within one experiment were presented in a single block, which consisted of 320 randomly intermixed trials in each of the following conditions: facing right and facing left (L or R) when upright, paired with either the same, or a mirror-reflected version, presented in each of the four orientations $\left(0^{\circ}, 90^{\circ}, 180^{\circ}\right.$ and $\left.270^{\circ}\right)$. This yielded a total of $2 \times 2 \times 4$ conditions with 20 trials in each condition.

Participants sat in front of a 17" computer screen (refresh rate $75 \mathrm{~Hz}$ ) placed at a distance of $120 \mathrm{~cm}$. 

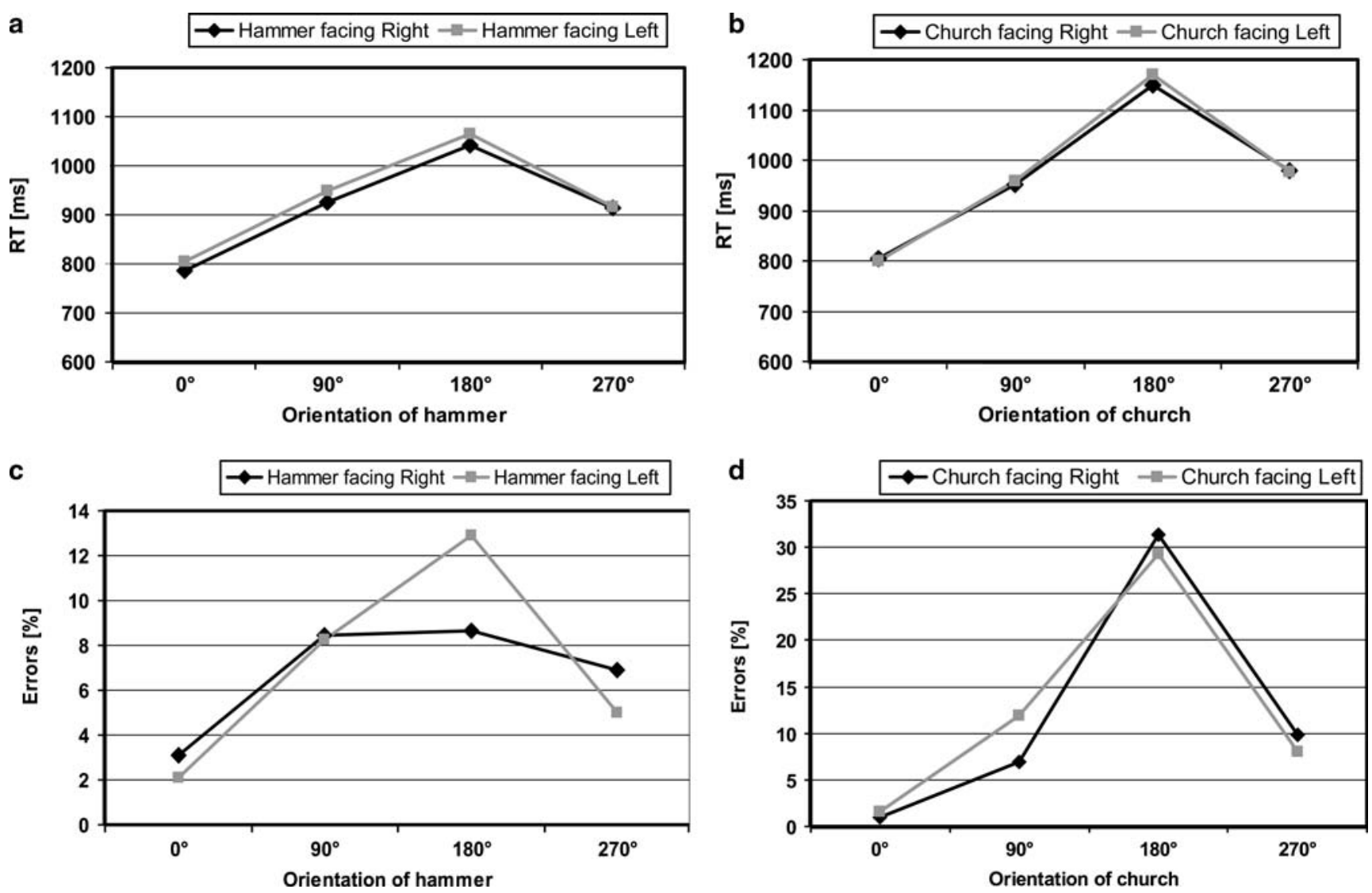

Fig. 2 Mean RT and mean percent error as function of orientation, plotted separately for the hammer $(\mathbf{a}, \mathbf{c})$ facing Right and Left and the church (b, d) facing Right and Left

Following electrode placement and completion of the handedness questionnaire, subjects were given instructions concerning the task, along with an illustration of the stimulus in various angles of rotation. At the beginning of the block, they had ten practice trials. They were instructed to avoid tilting their head, to keep their gaze on the fixation point throughout the task and were asked to answer as quickly as possible. For half the subjects, the response was given by pressing one key with their right index finger if the target and stimulus were facing the same direction and a second key with their middle finger if they were facing opposite directions. The other half of the group responded in the reverse manner. Similarly, half of the subjects started with the test trials, the other half with the control trials.

\section{EEG acquisition and analysis}

EEG recordings were performed in an electrically and noise shielded chamber and data acquisition was carried out continuously throughout the experiment. The EEG was acquired with a Geodesics Netamps system (Electrical Geodesics, Inc., USA) from 123 scalp electrodes (impedances $<50 \mathrm{k} \Omega$; vertex reference; $500 \mathrm{~Hz}$ digitization; band pass filtered $0.1-200 \mathrm{~Hz}$ ).

To calculate the ERP, epochs of EEG from $200 \mathrm{~ms}$ pre-stimulus to $600 \mathrm{~ms}$ post-stimulus onset were averaged for each of the eight stimulus conditions (two left-right $\times$ four orientations of the second stimulus) and from each subject. Only trials leading to correct responses were included. Off-line processing of the scalp data consisted of visual rejection of trials contaminated by artefacts and interpolation of bad channels using a spherical spline algorithm. Then, for each subject, the ERP was further down-sampled to a 111channel montage because the lowest line of electrodes was too often contaminated with noise or muscular artefacts. ERPs were band pass filtered $(1-40 \mathrm{~Hz})$, and recalculated against the average reference. When group averages (grand mean ERPs) were calculated, the individual ERPs were normalised to their mean global field power to eliminate general amplitude differences between subjects. However, no normalisation was performed for the statistical analysis with the individual data.

The grand mean ERPs for each of the eight conditions were computed over subjects. There were eight stimulus conditions, with four orientations $\left(0^{\circ}, 90^{\circ}, 180^{\circ}\right.$ and $270^{\circ}$ ) facing Right and the same four orientations facing Left, which lead to the following series: "R0", "R90", "R180", "R270", "L0", "L90", "L180" and "L270". For the test stimulus, the hammer, $0^{\circ}$ and $270^{\circ}$ of both left and right orientation represented the natural grip positions, the other four conditions represented the awkward grip for right-handers (see Fig. 1a). 
The recording of the electric activity from many different sites simultaneously allows the construction of topographic maps of the momentary electric activity on the scalp as well as the study of the time series of these maps with a millisecond resolution. ERP mapping attempts to determine points in time when map configurations change and/or when they differ between experimental conditions, which contrasts with the traditional analysis of ERP waveforms at certain electrode positions. The ERP mapping approach relies on the assumption that whenever the spatial configuration of the electric field on the scalp differs, different neuronal populations are active in the brain, reflecting an alteration of the global functional state of the brain (Lehmann 1986; Brandeis and Lehmann 1986; Michel et al. 1999, 2001, 2004a).

To define stimulus-specific topographic maps, a spatial cluster analysis (Pascual-Marqui et al. 1995) first identified the most dominant scalp topographies appearing in the group-averaged ERPs from each condition over time. This pattern analysis made it possible to summarise ERP data by a limited number of scalp configurations, the optimal number being determined by a modified cross validation criterion (Pascual-Marqui et al. 1995). The spatial correlation between the original maps of the grand mean ERPs and the cluster maps was then calculated which allowed us to determine the time segments during which each of these cluster maps were present (Michel et al. 1999, 2004a). Each segment is thought to represent a given "functional microstate" of the brain or a given computational step during information processing (Lehmann and Skrandies 1980; Michel et al. 1999), comparable to the traditional definition of ERP components.

This segmentation procedure allowed us to identify time periods during which the different stimulus conditions differ in terms of evoked scalp potential maps. In order to confirm these findings statistically, we then fitted the cluster maps that were found during a given time period to the individual ERPs of all subjects and all conditions. That means that for each time point of the individual subject's ERP, the scalp topography was compared to all segmentation maps and was labelled according to the one with which it best correlated. The results of the labelling revealed if a given ERP period in a given stimulus condition was more often described by one segmentation map versus another. Repeated measures ANOVA using stimulus condition and segmentation map as within-subject factors were used to determine significant differences in map presence (number of time frames).

Therefore, the spatio-temporal analysis procedure directly showed if different generator configurations better account for particular experimental conditions in particular time windows. If the processing of natural and awkward grips relies on distinct cortical networks, this analysis should reveal that different scalp topographies explain the ERPs of the different postures and orientations of the object. In contrast, no such differences should be found in the control condition.

\section{Source estimation analysis}

The final step of analysis consisted of estimating the localisation of the brain areas that differed during those segments where significantly different scalp potential maps were found between conditions. In these cases, a three-dimensional distributed linear inverse solution called LAURA (Grave de Peralta et al. 2001, 2004; Michel et al. 2004a) was calculated for the mean map of this time segment. A realistic head model based on the average brain provided by the Montreal Neurological Institute (MNI, Montreal, Canada) was used. Four thousand and twenty four equally spaced solution points (voxels) were defined within the grey matter of this averaged brain (for details see Grave de Peralta 2004). In cases where different maps explained the same time periods in different stimulus conditions, voxel-wise parametric mapping analysis was performed (Michel et al. 2004a; Murray et al. 2004). For that, the inverse solutions of the mean map over the corresponding time period was calculated for each subject and paired $t$-tests were calculated for each of the 4,024 solution points. To obtain an appropriate significance criterion for the statistical analysis, the correction for multiple tests was based on the number of independent measures. In EEG source imaging, this number corresponds to the number of electrodes on the scalp. Therefore, the $p$-values were corrected by the number of electrodes (111-electrode montage minus the reference $=110$ in this study) (Grave de Peralta et al. 2004; Michel et al. 2004a; Murray et al. 2004). This Bonferroni-type correction for multiple tests meant that only nodes with $p$-values $<.00045$ were considered significant.

ERP-analysis and the display of the inverse solutions were carried out with the in-house developed EEG analysis software package Cartool.

\section{Results}

\section{Behavioural results}

Response times and accuracy were recorded. Figure 2 shows the mean RTs for the correct responses (Fig. 2a, b) and error percentages (Fig. 2c, d) of all 13 subjects for the test and control conditions, respectively.

The RTs for the correct trials were analysed using a 2 $\times 4$ repeated measures ANOVA with Posture (right vs. left) and Orientation $\left(0^{\circ}, 90^{\circ}, 180^{\circ}, 270^{\circ}\right)$ as withinsubject factors. For the test stimulus, this analysis revealed a main effect of Orientation $(F(3,36)=46.8$, $p<.001$ ), with RTs increasing gradually as the stimulus was rotated further from the upright (Fig. 2a). Separate analyses for the right and left postures of the test stimulus were also carried out but did not yield any 
significant differences. Mental rotation rates were calculated for the hammer facing Right and facing Left, by fitting a regression line to the RT data in the respective conditions. The mean rate for the hammer facing Right was $709^{\circ} / \mathrm{s}$ and $689^{\circ} / \mathrm{s}$ for the hammer facing Left. In studies of the mental rotation of letters and digits, the average rate of mental rotation is typically around $400^{\circ}$ / s (Cooper and Shepard 1973). A possible explanation for the hammer being mentally rotated faster could be its familiarity and manipulability. However, these rates might not be very meaningful since the line was only fitted to three points $\left(0^{\circ}, 90^{\circ}, 180^{\circ}\right)$. Concerning error rates, a main effect of Orientation $(F(3,36)=5.63$, $p<.005)$ was found, with errors increasing gradually as the stimulus was rotated further from the upright. Trials in which no response was made within $3 \mathrm{~s}$ were discarded from the analysis of the behavioural data and not considered as errors. Figure $2 \mathrm{c}$ indicates a difference in postures of the hammer at 180 rotation angle, with fewer errors for "R180" than "L180"; however, this difference did not turn out to be significant $(t(12)=-1.74$, $p=.10)$.

The same analysis was performed for the control stimulus. Very similar results were obtained: For RTs a main effect of Orientation was found $(F(3,36)=50.5$, $p<.001)$, with RTs increasing gradually as the stimulus was rotated further from the upright. Again, no significant differences were found between right and left postures of the stimulus. Mental rotation rates were $523^{\circ} / \mathrm{s}$ and $490^{\circ} / \mathrm{s}$, respectively, for the church facing Right and facing Left. For error rates, a main effect of Orientation was also found $(F(3,36)=13.43, p<.001)$, with errors increasing gradually as the stimulus was rotated further from the upright.

\section{Electrophysiological results}

Analysis was performed separately for the test and the control experiment. For each, the grand mean ERPs of the eight stimulus conditions (two postures, four angles) were collectively subjected to the cluster analysis, which determined the optimal number and the spatial configuration of the most representative scalp potential maps. Using a cross validation criterion, eleven clusters (maps) were found to best explain the data of the test experiment with the hammer as stimulus, while seven maps were found to optimally explain the ERPs in the control experiment with the church as stimulus.

\section{Statistical analysis of the hammer's ERPS}

Fitting the 11 cluster maps to the 8 ERPs by means of spatial correlation indicated that they covered different time segments within and between the conditions (Fig. 3). For the statistical analysis, we divided the ERPs into three time periods, the first one covering the early latency components $(0-116 \mathrm{~ms})$, the second one the middle latency components (118-288 ms) and the third window covering the late components (290-680 ms). For each time window, the cluster maps that were found to be present in the grand mean data were searched in the individual ERP of each subject and condition.

In the first time window, four cluster maps were found in at least some of the eight grand mean ERPs. These four maps were fitted in the individual ERPs during this time period and each individual time point was labelled with the map it best correlated with. This gave for each subject the number of time points that each of the four cluster maps was found in each stimulus condition. The statistical analysis over subjects using ANOVAs with posture (2), rotation angle (4) and cluster maps (4) as repeated measures allowed us to determine which of the maps was systematically influenced by the stimulus variations in posture and/or angle. For the first time window, this overall ANOVA showed a significant angle $\times$ map interaction $(F(9,108)=3.94, p<.005)$. Subsequent ANOVAs for each map individually revealed highly significant effects of the factor "angle" for two maps (map N1: $F(3,36)=4.94, p<.005$; map A1: $F$ $=4.78, p<.006$ ). Figure $3 \mathrm{~b}$ shows that these two maps behave nearly opposite with respect to the rotation angle. Map N1 was more often present for $0^{\circ}$ and $270^{\circ}$ (the natural grips), while map A1 was more present for $90^{\circ}$ and $180^{\circ}$ (the awkward grips). In the grand mean data, these maps covered the time period between $\sim 60$ and $119 \mathrm{~ms}$.

In the second time window that included the prominent middle latency components $\mathrm{N} 2$ and $\mathrm{A} 2$, three cluster maps were present. The global posture $\times$ angle $\times$ maps ANOVA again revealed a significant angle $\times$ map interaction $(F(6,72)=5.46, p<.001)$. The individual ANOVAs for each map revealed again significant angle effects for two maps (map N2: $F(3,36)=6.99, p<.001$; map A2: $F(3,36)=6.69, p<.001)$. Again the maps behaved nearly opposite as is shown in Fig. 3b: map N2 is more present for $0^{\circ}$ and $270^{\circ}$, while map A2 is more present for $90^{\circ}$ and $180^{\circ}$. In the grand mean data, these two maps were found between 190 and $280 \mathrm{~ms}$, i.e., during the strong second ERP component (see Fig. 3a).

In the last window, the global ANOVA again revealed a highly significant map $\times$ angle interaction $(F(12,144)=2.97, p<.001)$. The individual ANOVAs for each of the five maps originally identified revealed significant angle effects for one map only (map R: $F(3,36)=4.35, p<.01$ ), appearing between 400 and $480 \mathrm{~ms}$ in the grand mean data. In contrast to the two earlier time windows, the duration of map $\mathrm{R}$ mirrored that of the RT, i.e., it increased from $0^{\circ}$ to $90^{\circ}$ to $180^{\circ}$ and then decreased for $270^{\circ}$ (Fig. $3 \mathrm{~b}$ ), reflecting the mental rotation process. In none of the three time windows were significant effects of the factor "posture" nor interactions with this factor found.

In summary, the analysis of the first and second time windows revealed differential responses for hammer positions that can readily be manipulated by righthanders as compared to those that are more awkward. In contrast, the last time window shows brain responses 


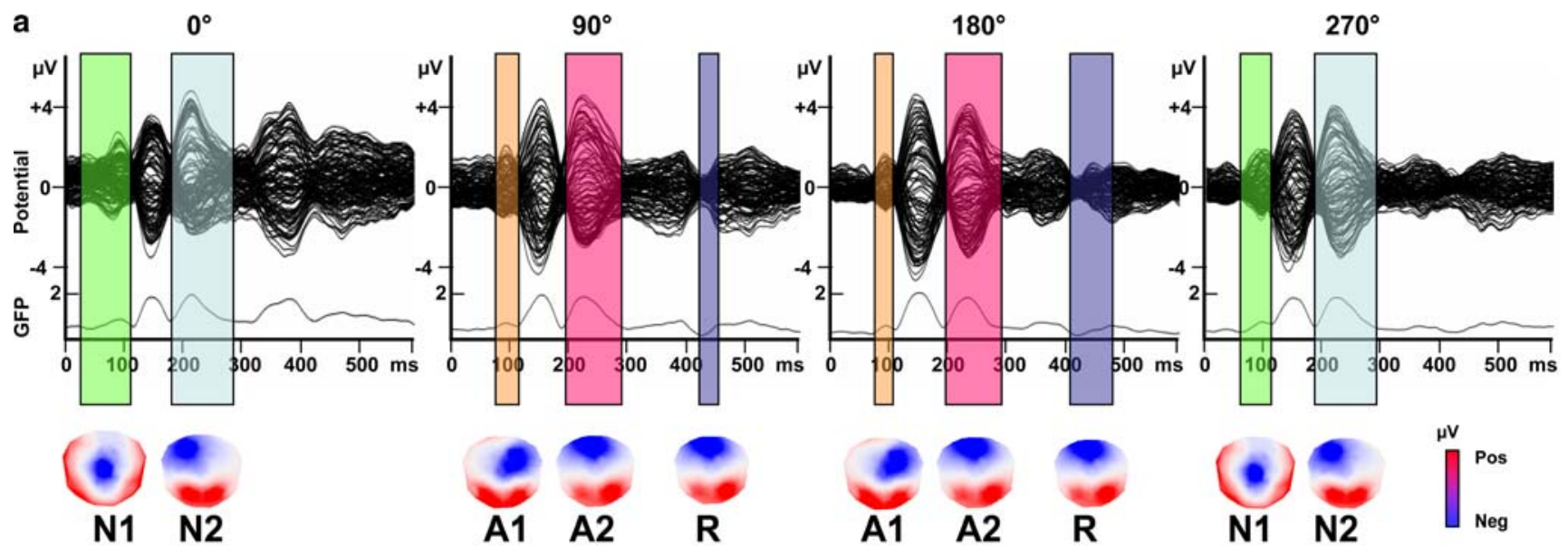

b ANGLE*MAP interaction: $F(3,36)=6.19, p<.001$

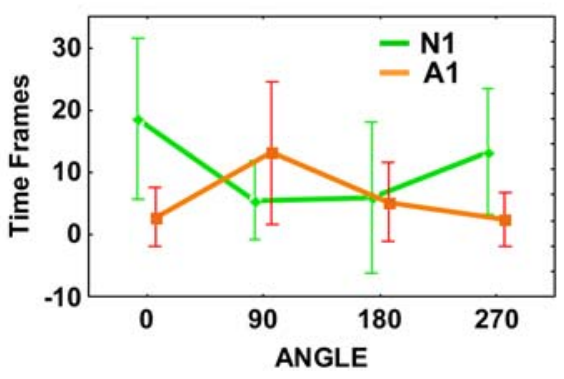

Fig. 3 a Overlapped traces of the grand mean ERPs for the four angles of rotation of the hammer (left and right postures averaged). The vertical colour bars indicate the time periods during which significantly different maps were found between the rotation angles. Maps N1 and A1 were on the average found between 60 and $118 \mathrm{~ms}$, covering the visual P100 component. Maps N2 and A2 were found between 190 and $280 \mathrm{~ms}$ and Map $\mathrm{R}$ was found between 420 and $480 \mathrm{~ms}$. The potential maps corresponding to these different time segments are given below the bars (blue $=$ negative, red $=$ positive potential, nose up, left ear left). b Results of the fitting procedure allowing statistical analysis of differences in the presence of the maps. Left: Map N1 is more present in the ERPs for $0^{\circ}$ and $270^{\circ}$ with significant interaction between angle and maps. Middle: Map N2 is more present in the ERPs for $0^{\circ}$ and $270^{\circ}$ while map A2 is more present for $90^{\circ}$ and $180^{\circ}$. Right: Map R increases its duration with increasing rotation angle from $0^{\circ}$ to $180^{\circ}$

that follow the RT, i.e., that increase with increasing rotation angle.

\section{Statistical analysis of the church's ERPS}

When fitting the seven cluster maps to the eight ERPs of the control experiment, no differences between conditions were found for the early stages of processing. However, a particular map appeared from $524 \mathrm{~ms}$ for rotated, but not upright stimuli. The statistical analysis of this late time window (290-680 ms) revealed an Orientation $\times$ Map interaction $(F(15,180)=3.28, p<.001)$. As for the hammer, the duration of this map (labelled map R) increased according to the angle of rotation. The duration of this map was inferred to represent the mental rotation process as its pattern perfectly matched the RTs. Thus, the only difference found for the control ERPs was a map that corresponded to the angle of rotation of the stimulus, but no differences were found between those two classes of orientations that revealed the early ERP differences in the test experiment with the hammer.

\section{Source localisation}

The distributed inverse solutions of the first significant time period of the test experiment $(60-116 \mathrm{~ms})$ revealed bilateral occipital sources in all conditions (i.e., awkward and normal grip postures of the hammer). Even though the scalp potential maps were significantly different between the conditions, as indicated by the map fitting analysis (Fig. 3b), the voxel-wise parametric mapping of the inverse solutions did not reveal significant differential activations (Fig. 4a). In contrast, significant differences were found for the second time period (184-288 ms). After Bonferroni correction using the number of electrodes as independent measures, significant activation differences were found in the left lateral fronto-parietal cortex with five neighbouring voxels being significantly more active for normal as compared to awkward positions (Fig. 4b). The Talairach coordinates extended from 47 to $53 \mathrm{~mm}$ in the inferior-superior direction, -13 to -19 in the posterior-anterior direction and -44 to -50 
Fig. 4 Sources estimated by the linear distributed source localisation algorithm LAURA for the two time segments where differences between normal and awkward grip postures of the hammer were found. The lowest row shows the voxels that significantly differed between the two conditions after Bonferroni-corrected voxel-wise parametric mapping of the activation in left motor areas was found for the normal oriented hammer in the second time window (184-288 ms)

Voxel-base

T-statistics (LAURA) inverse solutions. Increased

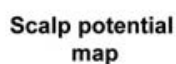

Source distribution

in the lateral-medial direction. This corresponds to the left hemisphere premotor/motor areas of the hand and digits. Thus, even though major activity was found in the left temporo-occipital areas in this time window, the significant difference between the two relevant orientations was in the motor cortex of the left hemisphere.

Localisation of map $\mathrm{R}$ indicated two major foci of activation in the test as well as in the control condition, one in the left temporo-occipital area and another in the right parietal lobe (Fig. 5).

\section{Discussion}

Behavioural results

This study investigated whether knowledge about the use of tools is taken into account during mental imagery. More specifically, we sought to determine the neural processes and temporal dynamics of brain activation associated with intrinsic manipulability, by investigating the mental rotation of a manipulable object. The behavioural results revealed a mental rotation function, with RTs (and errors) increasing gradually with the rotation angle from $0^{\circ}$ to $180^{\circ}$ and decreasing for $270^{\circ}$ for both the test and control stimuli. No significant difference was found between the two postures of the hammer (facing right and facing left).

Fig. 5 Sources estimated by the linear distributed source localisation algorithm LAURA for the mental rotation maps for both types of stimuli. Even though latency and scalp topography (polarity) differed between the two conditions, the maximal activity was found in right parietal areas as well as (somewhat weaker) in left temporo-occipital areas
Time window 60-160 ms

A1
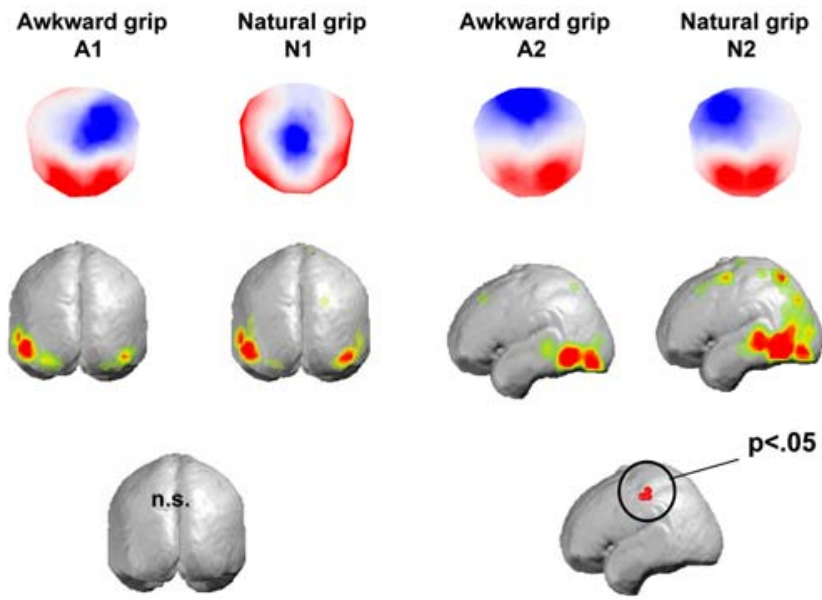

Time window 184-288 ms

ERP results

The electrophysiological results showed that even at a very early stage (between 0 and $116 \mathrm{~ms}$ post-stimulus onset) and then at a later stage (between 118 and $288 \mathrm{~ms}$ ) certain maps were more present for $0^{\circ}$ and $270^{\circ}$ orientations of the hammer, while others were predominant for $90^{\circ}$ and $180^{\circ}$ orientations. These maps appear to be related to the manipulability of the tool. Maps N1 and N2 appeared for the orientations representing Natural grips for right-handers (conditions "R0", "R270", "L0" and "L270") whereas the other maps A1 and A2 were present for the orientations that represent Awkward grips for right-handers (conditions "R90", "R180", "L90" and "L180"). This finding is consistent with there being stored associations between visual characteristics and specific actions and corroborates the findings of Tucker and Ellis (2004) in off-line tasks, who found that object affordance effects reflect the activation of stored action knowledge. Moreover, map N2 yielded a significantly increased activation in the areas of the left premotor/motor areas corresponding to the area of the representation of the hand, as indicated by Talairach coordinates (for example, Indovina and Sanes 2001). Thus, the manipulability of the object, reflected by this premotor/motor activation, is accessed at an early stage and prior to the mental rotation. One could speculate that in the case of tools presented in a natural grip, the activation of stored action knowledge built up from a
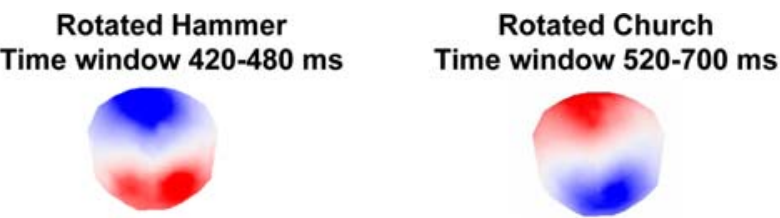

Source distribution
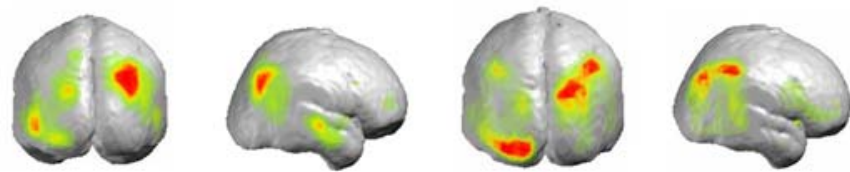
history of past interactions that have been integrated with the object representation itself, is facilitated compared to the presentation of the hammer in non-manipulable, awkward, orientations (Tucker and Ellis 2004).

As mentioned in the introductory section, Chao and Martin (2000) found that viewing and naming pictures of tools selectively activated the left ventral premotor cortex. Studies in monkeys have shown that neurons in the rostral part of the ventral premotor cortex (area F5) respond to the visual presentation of graspable objects, even in the absence of any subsequent motor activity (Jeannerod et al. 1995). Thus, the left ventral premotor region that responded selectively to tools in the Chao and Martin study may be the human homologue of the monkey F5 area. Viewing and naming tools also selectively activated the left posterior parietal cortex compared to naming other categories of objects. This response is similar to the firing of monkey AIP neurons to the visual presentation of graspable objects (Sakata et al. 1995). Rumiati et al. (2004) also identified two regions, which were activated when producing a wide range of skilled actions triggered by objects (controlled for perceptual, motor, semantic and lexical effects): left dorsal inferior parietal cortex and ventral inferior parietal cortex. The latter has been reported previously in association with tool use and in particular with grasping (Binkofski et al. 1999; Grafton et al. 1996; Grefkes et al. 2002). Thus, there seems to be a network of areas in the left hemisphere, which is implicated in the processing of tools and their associated actions: ventral premotor cortex, posterior middle temporal gyrus and intraparietal sulcus (Kellenbach et al. 2003).

Moreover, in their PET study, Grèzes and Decety (2002) investigated the involvement of motor representation during the visual perception of objects. This perception of man-made objects activated the occipitotemporal junction, inferior parietal lobule, SMA, inferior frontal gyrus, dorsal and ventral precentral gyrus in the left hemisphere, consistent with the idea that motor representations take part during the perception of objects. This advantage for the left hemisphere in tool representation is in accordance with evidence from neuropsychological studies of patients suffering from apraxia, which strongly implicate the left hemisphere in skilful object use. Our result of the source analysis also indicates activation of left ventral motor areas for hand-made objects, but only when they are perceived in natural grips. The Talairach coordinates point more specifically to the primary sensory-motor area of the hand than to the premotor areas. However, the limited spatial resolution of inverse solutions does not allow us to be certain whether premotor or primary motor areas are more involved.

This localisation is in line with Grafton et al. (1997) who found that the observation of real tools of common use activated the left dorsal premotor cortex in an area where arm/hand movements are represented and also with Grèzes et al. (2003) who showed activation of parietal and premotor areas when subjects observed manipulable objects. Thus, premotor activation in response to tool presentation, in the absence of any motor request, strongly resembles the activation of area F5 in the monkey where canonical neurons become active to the mere presentation of graspable objects (Rizzolatti et al. 1988; Murata et al. 1997). Moreover, these latter studies and our present findings revealed concomitant activations of parietal and premotor areas during passive observation of manipulable objects, which seem to correspond to the network found in monkeys, namely the parietal area AIP and the premotor area F5 (Murata et al. 1997). An interesting element to note though is that Grèzes et al. (2003) did not present tools but manipulable cylinders to their subjects and found similar activations. Thus, motor programs are not exclusively associated with tools but rather with any manipulable object. This is in agreement with the present findings in that the normal (manipulable) grips led to a significantly stronger activation of the fronto-parietal network than the awkward (non-manipulable) ones. What seems to matter then is not so much the fact that the object is a tool or not but that it is a graspable object. In order to categorise an object, one not only needs the description of its visual characteristics but also to understand its use. Thus, the "normal use" of an object is part of its overall semantic representation. The identification of a visual stimulus as some sort of graspable item would therefore expect a spreading of activation to information related to that object. The activations found in the present study may subserve the motoric aspects of the object's semantics. Thus, the object's motor program would be considered as part of the semantic knowledge about the object, in this case a hammer, in a manner similar to its association with nails for example. If the motor programs are considered as being part of the semantic representation of the object then one can suppose them to be automatically activated despite the fact that no interaction is required with the object presented. An element that supports this idea is that the motor activation appears early in the processing.

Indeed, one important result to come out of this study is the early timing of this motor cortex activation (184-288 ms). This activation appeared before mental rotation was performed ( $\sim 400-480 \mathrm{~ms})$. In addition, there was another, even earlier difference between normal and awkward positions (60-119 ms), i.e., during the P100 component. The source analysis revealed the expected activation of bilateral extrastriate areas during this component (Di Russo et al. 2002). Unfortunately, the distributed inverse solution was not sensitive enough to reveal significant activation differences for this first period even though the scalp potential maps were significantly different. This makes it difficult to speculate about brain areas that were differently activated during this early time period. However, a very early differential response to different classes of stimulus features is not astonishing and might indicate fast feed-forward coarse visual analysis (Thorpe et al. 1996; Bullier 2001; Michel et al. in press). 
Finally, we identified a specific map $(\mathrm{R})$ in the latest stage of processing, in both the test and control experiment, which was only present for rotated, but not upright, stimuli and whose duration clearly increased with the angle of rotation (see Fig. 3b). Moreover, the pattern of map R perfectly matched the RTs, which supports the assumption that it represents the mental rotation process. For the hammer, this segment occurred between 420 and $480 \mathrm{~ms}$, which is consistent with the findings of other mental rotation studies using alphanumeric characters (Harris and Miniussi 2003; Pegna et al. 1997; Peronnet and Farah 1989; Yoshino et al. 2000). For the church, the mental rotation map occurred around $100 \mathrm{~ms}$ later, probably due to the additional time required to perform the task with a more complex object.

The localisation of brain sources for map $\mathrm{R}$ indicated left occipito-temporal and (stronger) right parietal activations for both types of stimuli (Fig. 5), even though the scalp potential maps differed between them. Both activations are in full agreement with previous studies, which have shown that a cortical network including occipital, temporal and parietal lobes plays a role in mental rotation (Carpenter et al. 1999; Richter et al. 1997). Moreover, most imaging studies on mental rotation of objects have observed the involvement of the right parietal lobe (Pegna et al. 1997; Harris et al. 2000; Harris and Miniussi 2003; Zacks et al. 2003).

In conclusion, we have found evidence that implicit motor knowledge enters into the structure of visual perception of tools even in the cases when no movement is being executed, planned or intended. Moreover, there seem to be brain processes related to the manipulability of the tool. This early identification is consistent with the concept of affordance as more natural grips are processed differently than awkward orientations. Activation of stored representations of associations between the tool and the motor actions related to it is facilitated when the tool is presented in graspable orientations. In a broader framework, we suggest that the motor programs associated with an object are part of that object's semantic representation and that they are automatically activated regardless of the interaction with that object.

Acknowledgements This research was supported by an iMURS Scholarship and the Postgraduate Research Fund from Macquarie University to L. Petit. I. Harris was supported by an ARC Postdoctoral Fellowship. We thank Denis Brunet for the development of the software package Cartool that was used for this analysis, and Rolando Grave de Peralta for the development of the inverse solution algorithm LAURA. We also wish to thank Dr. Jeff Hamm and an anonymous reviewer for their useful comments and suggestions on an earlier draft of this manuscript.

\section{References}

Binkofski F, Buccino G, Stephan KM, Rizzolatti G, Seitz RJ, Freund HJ (1999) A parieto-premotor network for object manipulation: evidence from neuroimaging. Exp Brain Res $128: 210-213$
Brandeis D, Lehmann D (1986) Event-related potentials of the brain and cognitive processes: approaches and applications. Neuropsychologia 24:151-168

Bullier J (2001) Integrated model of visual processing. Brain Res Rev 36:96-107

Carpenter PA, Just MA, Keller TA, Eddy W, Thulborn K (1999) Graded functional activation in the visuospatial system with the amount of task demand. J Cogn Neurosci 11:9-24

Chao LL, Martin A (2000) Representation of manipulable manmade objects in the dorsal stream. Neuroimage 12:478-484

Chao LL, Haxby JV, Martin A (1999) Attribute-based neural substrates in posterior temporal cortex for perceiving and knowing about objects. Nat Neurosci 2:913-919

Cooper LA, Shepard RN (1973) Chronometric studies of the rotation of mental images. In: Chase WG (ed) Visual information processing. Academic Press, New York

Craighero L, Fadiga L, Umilta C, Rizzolatti G (1996) Evidence for visuomotor priming effect. Neuroreport 8:347-349

De'Sperati C, Stucchi N (1997) Recognizing the motion of a graspable object is guided by handedness. Neuroreport 8:27612765

Di Russo F, Martinez A, Sereno MI, Pitzalis S, Hillyard SA (2002) Cortical sources of the early components of the visual evoked potential. Hum Brain Mapp 15:95-111

Gibson JJ (1979) The ecological approach to perception. Houghton Mifflin, Boston

Grafton ST, Fagg AH, Woods RP, Arbib MA (1996) Functional anatomy of pointing and grasping in humans. Cereb Cortex 6:226-237

Grafton ST, Fadiga L, Arbib MA, Rizzolatti G (1997) Premotor cortex activation during observation and naming of familiar tools. Neuroimage 6:231-236

Grave de Peralta R, Gonzalez SL, Lantz G, Michel CM, Landis T (2001) Noninvasive localization of electromagnetic epileptic activity. Method descriptions and simulations. Brain Topogr 14:131-137

Grave de Peralta R, Menendez R, Murray MM, Michel CM, Martuzzi R, Gonzalez Andino SL (2004) Electrical neuroimaging based on biophysical constraints. Neuroimage 21(2):527-539

Grefkes C, Weiss PH, Zilles K, Fink GR (2002) Crossmodal processing of object features in human anterior intraparietal cortex: an fMRI study implies equivalences between humans and monkeys. Neuron 35:173-184

Grèzes J, Decety J (2002) Does visual perception of object afford action? Evidence from a neuroimaging study. Neuropsychologia 40:212-222

Grèzes J, Tucker M, Armony J, Ellis R, Passingham RE (2003) Objects automatically potentiate action: an fMRI study of implicit processing. Eur J Neurosci 17:2735-2740

Harris IM, Miniussi C (2003) Parietal lobe contribution to mental rotation demonstrated with rTMS. J Cogn Neurosci 15(3):315323

Harris IM, Egan GF, Sonkkila C, Tochon-Danguy HJ, Paxinos G, Watson JD (2000) Selective right parietal lobe activation during mental rotation: a parametric PET study. Brain 123(Pt 1):65-73

Indovina I, Sanes JN (2001) On somatotopic representation centers for finger movements in human primary motor cortex and supplementary motor area. Neuroimage 13:1027-1034

Jeannerod M, Arbib MA, Rizzolatti G, Sakata H (1995) Grasping objects: the cortical mechanisms of visuomotor transformation. Trends Neurosci 18(7):314-320

Kellenbach ML, Brett M, Patterson K (2003) Actions speak louder than functions: the importance of manipulability and action in tool representation. J Cogn Neurosci 15(1):30-46

Lehmann D (1986) Principles of spatial analysis. In: Gevins AS, Rémond A (eds) Handbook of electroencephalography and clinical neurophysiology, vol 1: methods of analysis of brain electrical and magnetic signals. Amsterdam, pp 309-354

Lehmann D, Skrandies W (1980) Reference-free of identification of components of checkerboard-evoked multichannel potential fields. Electroencephalogr Clin Neurophysiol 48(6):609-621 
Michel CM, Seeck M, Landis T (1999) Spatiotemporal dynamics of human cognition. News Physiol Sci 14(5):206-214

Michel CM, Thut G, Morand S, Khateb A, Pegna A, Grave de Peralta R, Gonzalez S, Seeck M, Landis T (2001) Electric source imaging of human brain functions. Brain Res Rev 36(23):108-118

Michel CM, Murray MM, Lantz G, Gonzalez S, Spinelli L, Grave de Peralta R (2004a) EEG source imaging. Clin Neurophysiol 115:2195-2222

Michel CM, Seeck M, Murray MM (2005) The speed of visual cognition. In: Hallett M, Phillips L, Schomer D, Mark J (eds) Advances in clinical neurophysiology, vol 57 (in press)

Murata A, Fadiga L, Fogassi L, Gallese V, Raos V, Rizzolatti G (1997) Object representation in the ventral premotor cortex (area F5) of the monkey. J Neurophysiol 78:2226-2230

Murray MM, Michel CM, Grave de Peralta R, Ortigue S, Brunet D, Gonzalez Andino S, Schnider A (2004) Rapid discrimination of visual and multisensory memories revealed by electrical neuroimaging. Neuroimage 21:125-135

Oldfield RC (1971) The assessment and analysis of handedness: the Edinburgh inventory. Neuropsychologia 9:97-113

Parsons LM (1987) Imagined spatial transformations of one's hands and feet. Cogn Psychol 19:178-241

Pascual-Marqui RD, Michel CM, Lehmann D (1995) Segmentation of brain electrical activity into microstates: model estimation and validation. IEEE Trans Biomed Eng 42(7):658665

Pegna AJ, Khateb A, Spinelli L, Seeck M, Landis T, Michel CM (1997) Unravelling the cerebral dynamics of mental imagery. Hum Brain Mapp 5:410-421

Peronnet F, Farah MJ (1989) Mental rotation: an event-related potential study with a validated mental rotation task. Brain Cogn 9:279-288

Petit LS, Harris IM (2005) Anatomical limitations in mental transformations of body parts. Vis Cogn 12(5): 737-758
Petit LS, Pegna AJ, Mayer E, Hauert CA (2003) Representation of anatomical constraints in motor imagery: mental rotation of a body segment. Brain Cogn 51:95-101

Richter W, Ugurbil K, Georgopoulos A, Kim SG (1997) Timeresolved fMRI of mental rotation. Neuroreport 8:3697-3702

Rizzolatti C, Camarada R, Fogassi L, Gentilucci M, Luppion G, Matelli M (1988) Functional organization of inferior area 6 in the macaque monkey. Area F5 and the control of distal movements. Exp Brain Res 71:491-507

Rumiati RI, Weiss PH, Shallice T, Ottoboni G, Noth J, Zilles K, Fink GR (2004) Neural basis of pantomiming the use of visually presented objects. Neuroimage 21:1224-1231

Sakata H, Taira M, Murata A, Mine S (1995) Neural mechanisms of visual guidance of hand action in the parietal cortex of the monkey. Cereb Cortex 5:429-438

Taira M, Mine S, Georgopoulos AP, Murata A, Sakata H (1990) Parietal cortex neurons of the monkey related to the visual guidance of hand movements. Exp Brain Res 83:29-36

Thorpe S, Fize D, Marlot C (1996) Speed of processing in the human visual system. Nature 381:520-522

Tucker M, Ellis R (1998) On the relations between seen objects and components of potential actions. J Exp Psychol Hum Percept Perform 24(3):830-846

Tucker M, Ellis R (2004) Action priming by briefly presented objects. Acta Psychol 116:185-203

Viviani P, Stucchi N (1992) Motor-perceptual interactions. In: Stelmach GE, Requin J (eds) Tutorials in motor behavior II. Elsevier Science, Amsterdam, pp 229-248

Warrington EK, Shallice T (1984) Category specific semantic impairments. Brain 107(3):829-854

Yoshino A, Inoue M, Suzuki A (2000) A topographic electrophysiologic study of mental rotation. Cogn Brain Res 9:121-124

Zacks JM, Gilliam F, Ojemann JG (2003) Selective disturbance of mental rotation by cortical stimulation. Neuropsychologia 41:1659-1667 